Lizano Araya, MA. y Lizano Rodríguez, O. (2020): "Escenarios ante el aumento del nivel del mar por cambio climático para la localidad del Cocal, Puntarenas, Costa Rica", GeoFocus, revista Internacional de Ciencia y Tecnología de la Información Geográfica, 26, 3-20. http://dx.doi.org/10.21138/GF.656

\title{
ESCENARIOS ANTE EL AUMENTO DEL NIVEL DEL MAR POR CAMBIO CLIMÁTICO PARA LA LOCALIDAD DEL COCAL, PUNTARENAS, COSTA RICA
}

\author{
${ }^{a}$ MELVIN ARNOLDO LIZANO ARAYA (D) Y 'OMAR GERARDO LIZANO RODRIGUEZ \\ Universidad de Costa Rica \\ Ciudad Universitaria Rodrigo Facio Brenes, San José, San Pedro, Costa Rica \\ amelvin.lizanoaraya@ucr.ac.cr, ${ }^{\mathrm{b}}$ omarglizano@gmail.com
}

\section{RESUMEN}

Se analiza el aumento del nivel del mar tomando como base el nivel promedio de mareas altas para la región Pacífico de Costa Rica. Este estudio se realizó a partir de modelos, observaciones y mediciones efectuadas en campo, que presentan un nivel del mar y su respectivo ascenso para cuatro escenarios de acuerdo con los siguientes años: 2030, 2050, 2070 y 2100. Se elaboran escenarios reales para simular el comportamiento del nivel del mar en los próximos 100 años para la localidad del Cocal de Puntarenas. Se estudian los niveles de mareas astronómicas a partir de predicciones mareales. El aumento del nivel del mar en los próximos años por calentamiento global es, según los escenarios de Nerem et al. (2018), de alrededor $3 \mathrm{~mm}$ por año para la Costa del Pacífica en general y se utiliza como base para crear las distintas combinaciones y generar los niveles mareográficos representados en este artículo.

Palabras clave: nivel de mareas; escenarios; cambio climático; modelos; topografía y batimetría.

SCENARIOS BEFORE THE RISE IN SEA LEVEL, FOR THE TOWN OF COCAL, PUNTARENAS, COSTA RICA

\section{ABSTRACT}

The sea level rise is analyzed based on the average level of high tides for the Pacific region of Costa Rica. This study was carried out from models, observations and measurements made in the field, which present a sea level and its respective ascent for four scenarios according to the following years: 2030, 2050, 2070 and 2100. Real scenarios are elaborated to simulate the behavior of sea level in the next 100 years for the town of Cocal of Puntarenas. The levels of astronomical tides are studied from tidal predictions. The rise in sea level in the coming years due to global warming, is according to the Nerem et al. (2018), scenarios of around $3 \mathrm{~mm}$ per year for the Pacific Coast in general and is used as a basis to create the different combinations and generate the tide 
Lizano Araya, MA. y Lizano Rodríguez, O. (2020): "Escenarios ante el aumento del nivel del mar por cambio climático para la localidad del Cocal, Puntarenas, Costa Rica", GeoFocus, revista Internacional de Ciencia y Tecnología de la Información Geográfica, 26, 3-20. http://dx.doi.org/10.21138/GF.656

levels represented in this article.

Keywords: tidal level, scenarios, climate change, models, topography and bathymetry.

\section{Introducción}

Dentro del Sistema Tierra los efectos de las actividades humanas pueden detectarse a escala planetaria. Estos efectos han llevado a los científicos a definir una nueva época geológica, el Antropoceno, con base en evidencias que muestran que los procesos atmosféricos, geológicos, hidrológicos y biológicos del Sistema Tierra entre otros, están siendo alterados por las actividades humanas (PNUMA, 2013). Los cambios más fácilmente reconocidos incluyen el incremento de las temperaturas globales y de los niveles del mar, así como la acidificación de los océanos asociados al aumento en las emisiones de gases de efecto invernadero (PNUMA, 2013).

Lo anterior se refleja con un decremento en las masas glaciares, pero no en todas las regiones, así como una disminución de la cubierta de nieve en las regiones del hemisferio norte, los escurrimientos máximos de los glaciares y el derretimiento de la nieve se presentan más temprano en el año provocando la retirada de los glaciares, la fracturación de las grandes plataformas de hielo, el aumento del nivel del mar y el derretimiento del hielo permanente (PNUMA, 2013, 118). Todo esto es una influencia directa del aumento de la temperatura, expresado en el informe escrito por el IPCC $(2014,10)$, donde se apunta que es probable que el aumento de la temperatura media global en superficie a final del siglo XXI (2081-2100) respecto de $1986-2005$ sea de $0.3{ }^{\circ} \mathrm{C}$ a $1.7{ }^{\circ} \mathrm{C}$ bajo el escenario RCP2.6 y donde la región del Ártico seguirá calentándose más rápidamente que la media global. A la vez, el documento indica que es prácticamente seguro que se produzcan temperaturas extremas calientes más frecuentes y frías menos frecuentes en la mayoría de las zonas continentales, en escalas temporales diarias y estacionales, conforme vaya aumentando la temperatura media global en superficie.

La extensión de los hielos marinos árticos ha disminuido en 3,5\% y el $4.1 \%$ por decenio, con disminuciones estivales aún más acentuadas entre el $9,4 \%$ y el $13,6 \%$ por decenio (IPCC, 2014, 44). En promedio, los glaciares de montaña y la cubierta de nieve están decreciendo de manera acelerada, cambio que se ha manifestado en los últimos decenios, a tal punto que es probable que antes de mediados de siglo, el océano Ártico esté casi libre de hielo en el mes de septiembre, momento mínimo estival de hielo marino (IPCC, 2014, 12). Como indica Lambrechts $(2007,4)$ : "La extensión de la capa de nieve media mensual en el hemisferio norte ha disminuido a un ritmo de $1.3 \%$ por decenio en los últimos 40 años, registrándose las mayores pérdidas en la primavera y el verano, lo que supone reducciones en la capa de nieve".

A escala global se prevé la inundación de miles de kilómetros cuadrados de humedales costeros y tierras bajas (Bedia, 2004, 1). También es previsible el retroceso de playas y la ruptura de infraestructuras litorales de protección, la salinidad de acuíferos y la pérdida de tierras productivas para la agricultura y la ganadería (Bedia, 2004, 1). Los patrones de erosión y sedimentación litorales se verán modificados y afectarán instalaciones portuarias, enclaves turísticos y ecosistemas de especial valor, entre otras zonas sensibles (Van der Meulen et al., 1991, 106). Las consecuencias previsibles son el retroceso por los cambios en la dinámica litoral, como por la proliferación de 
Lizano Araya, MA. y Lizano Rodríguez, O. (2020): "Escenarios ante el aumento del nivel del mar por cambio climático para la localidad del Cocal, Puntarenas, Costa Rica", GeoFocus, revista Internacional de Ciencia y Tecnología de la Información Geográfica, 26, 3-20. http://dx.doi.org/10.21138/GF.656

estructuras de protección que alterará el ambiente costero en general (Bedia, 2004, 1). Es un hecho fundamental que todo lo anterior traerá grandes consecuencias que implicaran la intensificación del efecto de temporales marítimos y el aumento de la vulnerabilidad sobre las áreas costeras (Dawahidi, et al., 2019).

Asimismo, el modelo climático, elaborado por Otto-Bliesner et al. $(2006,1751)$ que concuerda con datos obtenidos de registros paleoclimáticos de testigos de hielo, polen fósil, sedimentos marinos y marcas químicas utilizados para comprobar la precisión del modelo. Se efectuó un cálculo del aumento para el año 2100, el cual indica que las costas del Sur, Suroeste y Surestes de Estado Unidos se verán seriamente afectadas, pues se registra un aumento aproximado de 6 metros del nivel del mar. Ciudades como Nueva Orleans desaparecerán para esa fecha, al igual que ciertas zonas de la Florida, como los cayos (Otto-Bliesner et al., 2006, 1752).

El ascenso del nivel del mar es una problemática que podría afectar al $50 \%$ de la población mundial que habita en las áreas costeras (Ramos et al, 2016). También se debe considerar la tendencia de concentración del calor en los océanos, que genera la expansión térmica. Lo anterior es una preocupación que tiene hoy por hoy la comunidad científica internacional y es dónde se debe centrar la atención del público en general. El aumento del calor en los océanos se estima en $(19,67 \pm 0.83) \times 1022 \mathrm{~J}$ para el año 2018 y que ocasionó un aumento del nivel medio del mar aproximadamente en $29.5 \mathrm{~mm}$ por encima de la media que se dio entre 1981-2010 y de 1.4 del 2017 (Cheng et al., 2019).

Costa Rica no escapa ante tal situación y principalmente, a lo que es el aumento del nivel de los océanos (Lizano, 1997, 172; Lizano y Salas, 2001, 172; Lizano, 2013, 9), debido a que al estar en un istmo y tener costas en ambas vertientes (Pacífica y Caribe) lo hace vulnerable ante futuros eventos de este tipo. Como lo indica Díaz $(1999,5)$, Costa Rica tiene más de $1100 \mathrm{~km}$ de línea de costa en el Océano Pacífico y más de $200 \mathrm{~km}$ en el Mar Caribe. Patrones de erosión desde leves, moderados y hasta severos ya se está viendo en las costas de Costa Rica, como lo señala Lizano (2013, 10), tanto en el Pacífico, como en el Caribe.

Para el caso de Costa Rica, se cuenta con el estudio efectuado por Díaz (1999), el cual determinó las zonas de riesgo ante un ascenso del nivel del mar: Punta Morales-Tárcoles. En este estudio Díaz utilizó la técnica AVVA (Análisis de Vulnerabilidad Asistido por Video-grabación Aérea, propuesta por Leatherman et al. (1995), y efectuó una clasificación del frente costero de acuerdo con su vulnerabilidad ante la acción de las olas o de los cambios del nivel del mar, y combinó ese método con el reconocimiento terrestre, el estudio estereoscópico de las aerofotos para trazar sobre ellas, los límites de regresión e inundación (transgresión). Es de resaltar que ese estudio se realizó con unos pocos datos topográficos aproximados alrededor de la Ciudad de Puntarenas, y sobre los cuales se sobrepusieron los escenarios del nivel del mar por calentamiento solamente. Nadie ha realizado escenarios de nivel del mar relativos a las demás componentes mareográficos, y más aún, refiriendo estos niveles al nivel "0" (cero) del mar o promedio de sicigias (Lizano, 2006, $53 ; 2009,19)$.

Otro de los estudios es el de Lizano y Lizano (2010) que es una buena primera aproximación a la generación de escenarios ante el aumento del nivel del mar y otras tres componentes oceanográficas para la ciudad de Puntarenas, que se toma como referencia base, al existir una carencia de este tipo de estudios para el país. 
Lizano Araya, MA. y Lizano Rodríguez, O. (2020): "Escenarios ante el aumento del nivel del mar por cambio climático para la localidad del Cocal, Puntarenas, Costa Rica", GeoFocus, revista Internacional de Ciencia y Tecnología de la Información Geográfica, 26, 3-20. http://dx.doi.org/10.21138/GF.656

\section{Materiales}

\subsection{El caso de estudio.}

Es la generación de escenarios de escenarios ante el aumento del nivel del mar para la zona del Cocal, Puntarenas, Costa Rica para los períodos del 2030, 2050, 2070 y 2100, que permita tomar acciones en el plano del ordenamiento territorial y la toma de decisiones a nivel nacional.

\section{Delimitación espacial.}

La comunidad del Cocal forma parte de la aguja litoral sobre la que se asienta la ciudad de Puntarenas y otras comunidades. Se ubica en una lengua de tierra de varios kilómetros de largo y unos $400 \mathrm{~m}$ de ancho, flanqueada por un estero hacia el Norte de la ciudad, y por el Océano Pacífico al Sur-Suroeste, por el otro. Puntarenas es la provincia más grande de Costa Rica y está comunicada con el resto del país por la vía interamericana. Se encuentra a $115 \mathrm{~km}$ (al Noroeste) de la ciudad de San José ver figura 1.

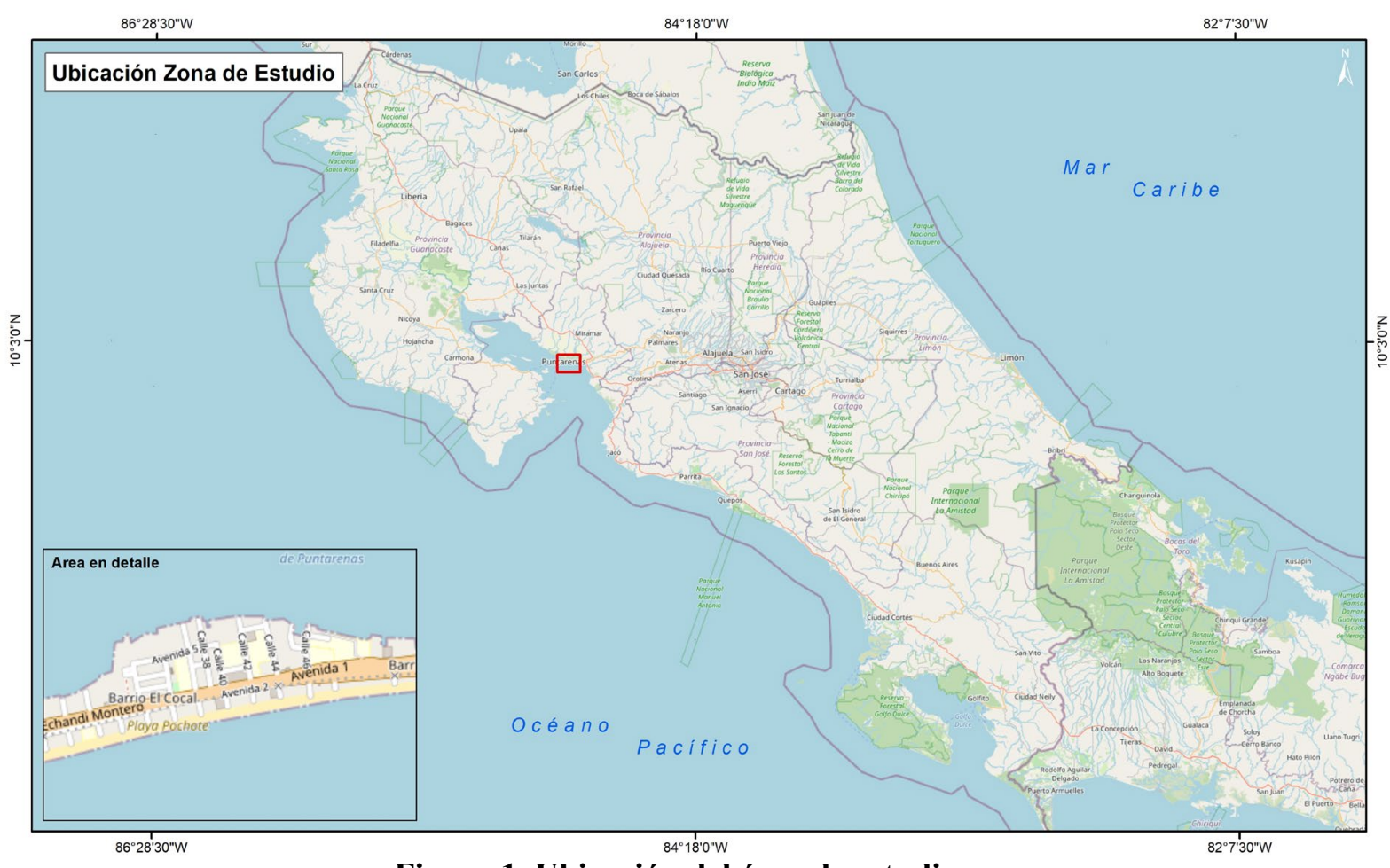

Figura 1. Ubicación del área de estudio.

En general, la provincia se caracteriza por tener un clima cálido y húmedo, del tipo tropical húmedo, con temperaturas máximas que rondan $\operatorname{los} 34^{\circ} \mathrm{C}$ y mínimas que rara vez bajan de $20^{\circ} \mathrm{C}$.

El área de estudio comprende un $16,15 \%$ del territorio de la provincia de Puntarenas, el cual está referenciado por las coordenadas métricas CRTM05 centrales $1103500 \mathrm{~m}$ falso norte y 
Lizano Araya, MA. y Lizano Rodríguez, O. (2020): "Escenarios ante el aumento del nivel del mar por cambio climático para la localidad del Cocal, Puntarenas, Costa Rica", GeoFocus, revista Internacional de Ciencia y Tecnología de la Información Geográfica, 26, 3-20. http://dx.doi.org/10.21138/GF.656

408500 m falso este, la altitud máxima donde se asienta la ciudad de Puntarenas es de 4 metros sobre el nivel medio del mar.

\section{Los datos.}

El levantamiento topográfico de la zona del Cocal se realizó mediante el sensor activo Lidar que deriva en una gran nube de puntos para ambas zonas permitiendo obtener modelos de elevación, de alta resolución espacial del orden de $50 \mathrm{~cm}$. Estos datos son facilitados por la Comisión Nacional de Emergencia (CNE) Consultado el 20 de Julio de 2020 (https://www.cne.go.cr/) de un sobrevuelo realizado para cubrir las costas del Pacífico y Caribe del país, entre los años 2015-2016. Al revisar y validar los datos Lidar de acuerdo con la clasificación internacional de la ASPRS se procede a verificar la calidad y en algunos casos, se tiene, que de la nube de puntos de ambas zonas entre un 30 y $40 \%$ de los datos de suelo, se encuentran en la categoría de no clasificados, por lo que se debió proceder a la fotointerpretación y corrección de estos para contar con un área totalmente densificada que permita la obtención de cartografía con un detalle menor a 1:1000.

Para la zona del Cocal se obtuvo posterior a la clasificación y fotointerpretación un total de 3510291 puntos de elevación que permite obtener el modelo de alta resolución espacial. La zona de cubrimiento de todo el Lidar se puede apreciar en la figura 2.

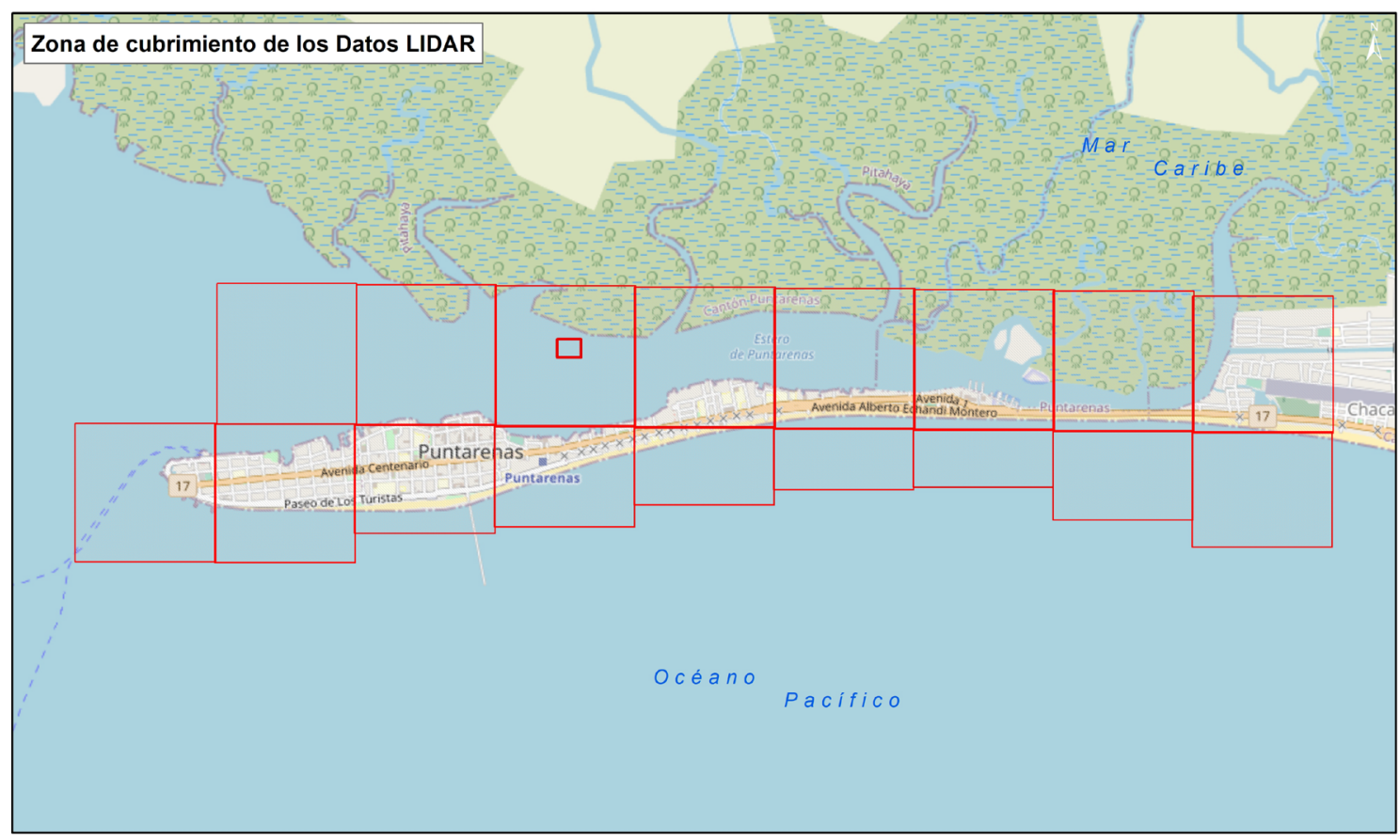

Figura.2. Cubrimiento datos lidar 
Lizano Araya, MA. y Lizano Rodríguez, O. (2020): "Escenarios ante el aumento del nivel del mar por cambio climático para la localidad del Cocal, Puntarenas, Costa Rica", GeoFocus, revista Internacional de Ciencia y Tecnología de la Información Geográfica, 26, 3-20. http://dx.doi.org/10.21138/GF.656

\subsection{Técnicas de análisis.}

Una vez con los datos filtrados, se procede a realizar el trabajo de campo, para realizar las mediciones del nivel del mar, y con el uso de las predicciones de marea para Puntarenas (Lizano, 2006, 53), se estable la diferencia de la marea con el nivel 0 de referencia (marea de sicigia). Luego se realiza el traslado a un punto terrestre conocido, obteniendo con ello un factor de ajuste respecto al punto de elevación según la siguiente fórmula:

$$
\text { FC }=\text { EPM }- \text { EPCT }
$$

FC: Factor de Corrección

EPM: Elevación Punto Marea

EPCT: Elevación Punto Control Terrestre

Al existir una carencia de un modelo Geoidal propio para el país al momento del estudio, se toma como referencia el modelo Geoidal WGS84 garantizando con ello la estandarización de toda la información sobre dicho geoide.

Con los datos obtenidos en el levantamiento de campo y los datos de topográficos obtenidos del lidar se procedió a unificar los niveles de referencia topográficos, de manera que se tuviera en una sola capa para realizar la modelización.

Este nivel 0 del mar (nivel de sicigias) al que se refirieron los datos de una tabla de mareas, fue establecido para el caso del Cocal sobre el Estero de Puntarenas. Se estableció un promedio entre el valor de los datos dados por el Lidar y los obtenidos en campo que sí tienen el nivel de referencia al 0 de la marea. La altura obtenida a partir de los datos de campo para la zona de Puntarenas es de $0.82 \mathrm{~m}$. Al comparar los puntos anteriores con los dados por el Lidar para la zona del Cocal, indica $1.50 \mathrm{~m}$, se tiene una diferencia absoluta para el primer punto de $0.68 \mathrm{~cm}$ entre cada uno de esos puntos, valor que se restó a los datos topográficos, con el fin de homologarlos al nivel "0" del mar, que corresponde al promedio de sicigias (Lizano, 2006, 53; 2009, 19).

Para trabajar la información de mareas astronómicas se utilizó el software Tidal \& Currents (Lizano, 2006, 53), cuyas predicciones incluidas en el programa, van desde el año 1901 hasta el año 2099.

Los distintos niveles del mar se modelaron utilizando un Sistema de Información Geográfica (SIG), asumiendo un aumento del nivel del mar entre $0.20 \mathrm{~cm}, 0.30 \mathrm{~cm}, 0.60 \mathrm{~cm}$ y 0.82 $\mathrm{m}$, según los criterios del informe del $\operatorname{IPCC}(2014,62)$ así como lo que muestra la tabla 1 he indicado por Nerem et al. (2018). Para el caso de estudio, al nivel de las mareas máximas para el Pacífico de Costa Rica (Lizano, 2006, 56) se le sumará cada uno de los incrementos mencionados, son conservadores no extremos. El máximo valor de marea para Puntarenas es $3.29 \mathrm{~m}$ (Lizano y Lizano, 2010, 223), y un nivel de marea de $3.0 \mathrm{~m}$ comienza a inundar paulatinamente el sector del muelle que corresponde al mercado de Puntarenas. 
Lizano Araya, MA. y Lizano Rodríguez, O. (2020): "Escenarios ante el aumento del nivel del mar por cambio climático para la localidad del Cocal, Puntarenas, Costa Rica", GeoFocus, revista Internacional de Ciencia y Tecnología de la Información Geográfica, 26, 3-20. http://dx.doi.org/10.21138/GF.656

Tabla 1. Aumento del Nivel del Mar para el Pacífico

\begin{tabular}{|ccc|}
\hline Marea Base & Año & Aumento \\
\hline $\mathbf{3}$ m & 2030 & 0.20 \\
\hline & 2050 & 0.30 \\
\hline & 2070 & 0.60 \\
\hline 3,29 m (Marea Máxima cada 4.65 años) & 2100 & 0.82 \\
\hline & 2030 & 0.20 \\
\hline & 2070 & 0.30 \\
\hline & 2100 & 0.60 \\
\hline
\end{tabular}

Fuente: Elaboración propia con datos IPCC (2014)

Aparte de la modelización con los datos de mareas, se utilizaron observaciones puntuales en la zona del Cocal con el fin de ajustar los datos de topografía al nivel "0" del mar o nivel de mareas de sicigias (Lizano, 2006, 53).

\section{Descripción y análisis de resultados.}

La altura topográfica promedio para la zona del Cocal es de $0.89 \mathrm{~m}$ referida al valor de desviación estándar de 3.30 que para la zona es lo esperable por el volumen y dispersión de datos, referidos al nivel 0 del mar, lo cual indica que el Cocal es una zona sumamente vulnerable a fenómenos meteorológicos y oceanográficos. Con estos datos se encontró que la máxima altura topográfica para los primeros 500 metros a partir de la costa, para la zona del Cocal es de $7.82 \mathrm{~m}$ a partir del nivel "0" como se aprecia en la figura 3. El punto de mayor elevación en el Cocal se encuentra sobre la línea férrea hacia el Sur de la comunidad, como se observa en la figura 3. 
Lizano Araya, MA. y Lizano Rodríguez, O. (2020): "Escenarios ante el aumento del nivel del mar por cambio climático para la localidad del Cocal, Puntarenas, Costa Rica", GeoFocus, revista Internacional de Ciencia y Tecnología de la Información Geográfica, 26, 3-20. http://dx.doi.org/10.21138/GF.656

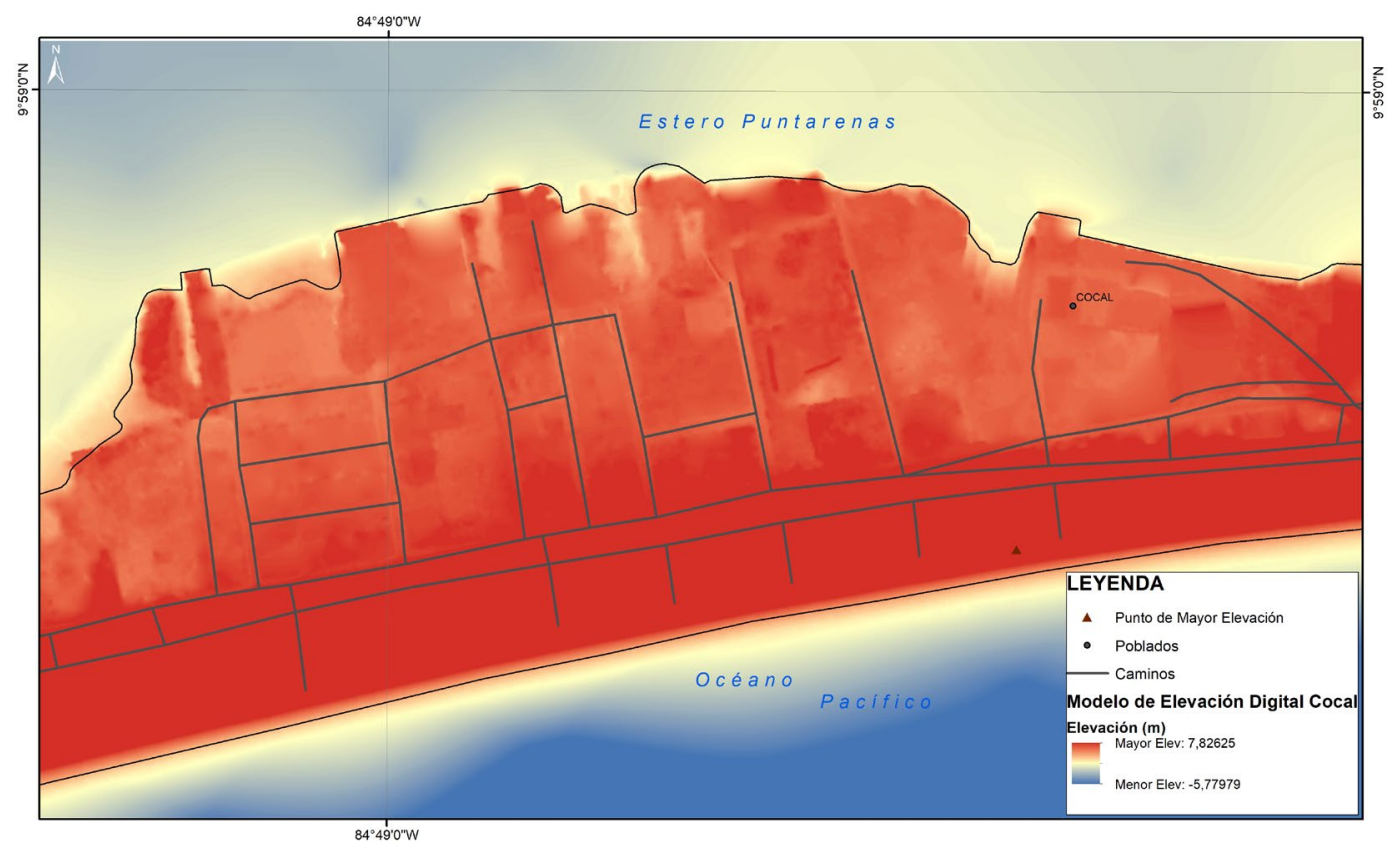

Fig. 3 Modelo de Elevación Cocal, Puntarenas

\section{Mareas máximas}

A partir de los datos mencionados en la tabla 1 se sabe que una marea máxima en Puntarenas es de $3.29 \mathrm{~m}$ (Lizano, 2006, 59). Se sabe que para Puntarenas mareas mayores a $2.95 \mathrm{~m}$, comienzan a inundar regiones de la Comunidad del Cocal, así como mareas mayores a $3.0 \mathrm{~m}$ comienzan a inundar las cercanías del Mercado Central, Banco Nacional y Banco de Costa Rica, como también la Comunidad del Cocal y algunos sectores de Barrio del Carmen. En la figura 4 se puede apreciar las áreas inundables en la zona del Cocal con mareas de $3.05 \mathrm{~m}$ y $3.29 \mathrm{~m}$. 
Lizano Araya, MA. y Lizano Rodríguez, O. (2020): "Escenarios ante el aumento del nivel del mar por cambio climático para la localidad del Cocal, Puntarenas, Costa Rica", GeoFocus, revista Internacional de Ciencia y Tecnología de la Información Geográfica, 26, 3-20. http://dx.doi.org/10.21138/GF.656
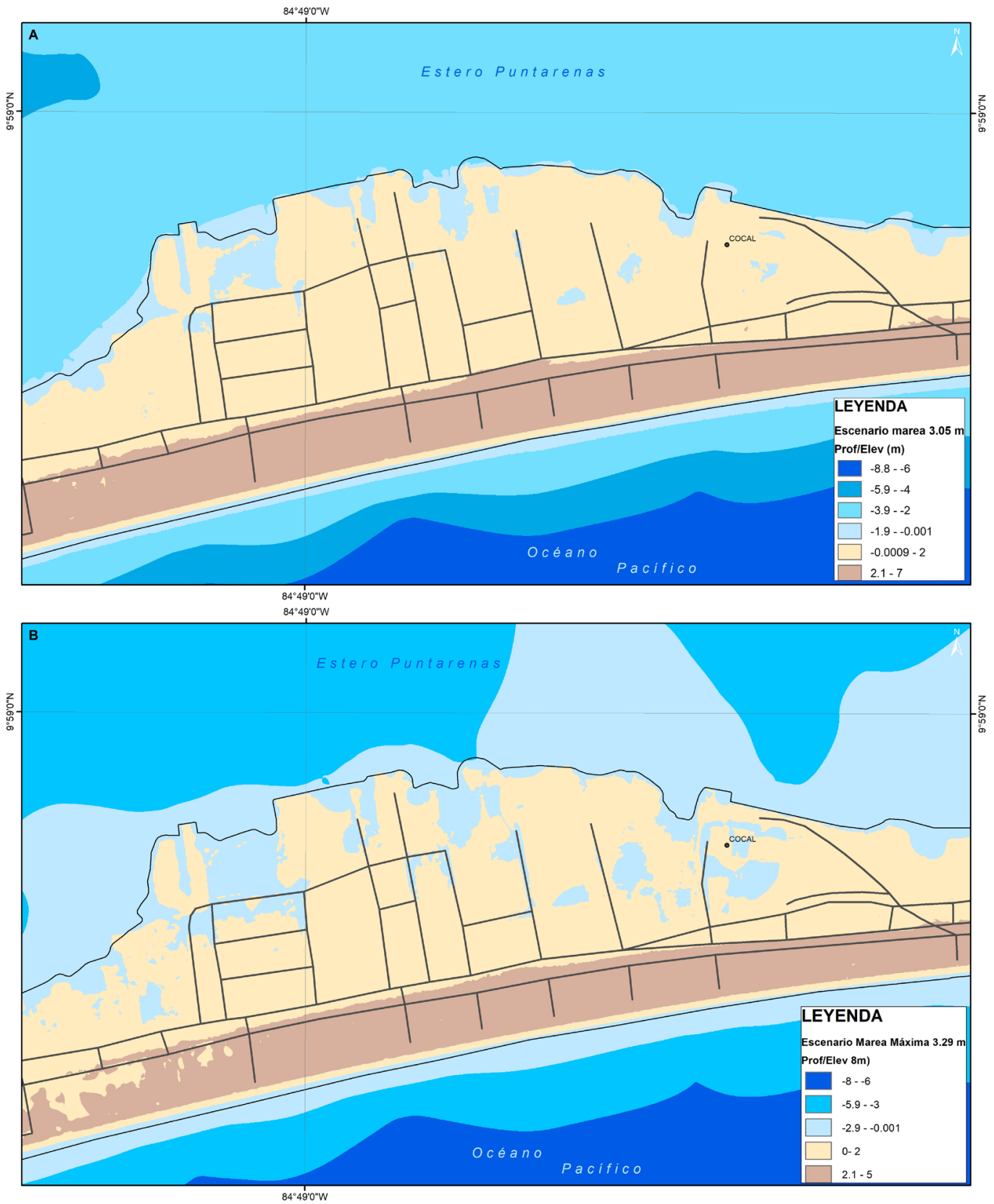

Fig.4. Nivel de inundación para el Cocal: mareas mayores a 3.05 m (A) y mareas máximas $3.29 \mathrm{~m}(\mathrm{~B})$.

Múltiples escenarios se podrían construir para ambas zonas a partir de los diferentes niveles de marea astronómica: nivel " 0 ", nivel promedio de referencia $(3 \mathrm{~m})$, etc., con las distintas combinaciones de las componentes mareográficos, en este caso, el aumento del nivel del mar. Lo importante entonces, son aquellos niveles ante el aumento del nivel del mar que tienen potencial de 
Lizano Araya, MA. y Lizano Rodríguez, O. (2020): "Escenarios ante el aumento del nivel del mar por cambio climático para la localidad del Cocal, Puntarenas, Costa Rica", GeoFocus, revista Internacional de Ciencia y Tecnología de la Información Geográfica, 26, 3-20. http://dx.doi.org/10.21138/GF.656

inundación para las zonas de estudio. Las combinaciones seleccionadas de estos niveles se resumen en la tabla 2.

Tabla 2: Niveles mareográficos utilizados como posibles escenarios ante el aumento del nivel del mar

\begin{tabular}{|c|c|c|c|c|}
\hline Escenario & $\begin{array}{c}\text { Marea } \\
\text { Base }\end{array}$ & Año & Aumento (m) & Marea Total (m) \\
\hline 1 & $3 \mathbf{~ m}$ & 2030 & 0.20 & 3.20 \\
\hline 2 & & 2050 & 0.30 & 3.30 \\
\hline 3 & & 2070 & 0.60 & 3.60 \\
\hline 4 & & 2100 & 0.82 & 3.82 \\
\hline 5 & $3.29 \mathrm{~m}$ & 2030 & 0.20 & 3.49 \\
\hline 6 & & 2050 & 0.30 & 3.59 \\
\hline 7 & & 2070 & 0.60 & 3.89 \\
\hline 8 & & 2100 & 0.82 & 4.12 \\
\hline
\end{tabular}

Las figuras 5 y 6, muestran los niveles de inundación para el sector del Cocal que pudieran generarse a partir de escenarios de mareas de $3.20 \mathrm{~m}$ y $3.30 \mathrm{~m}$ en la zona de estudio.

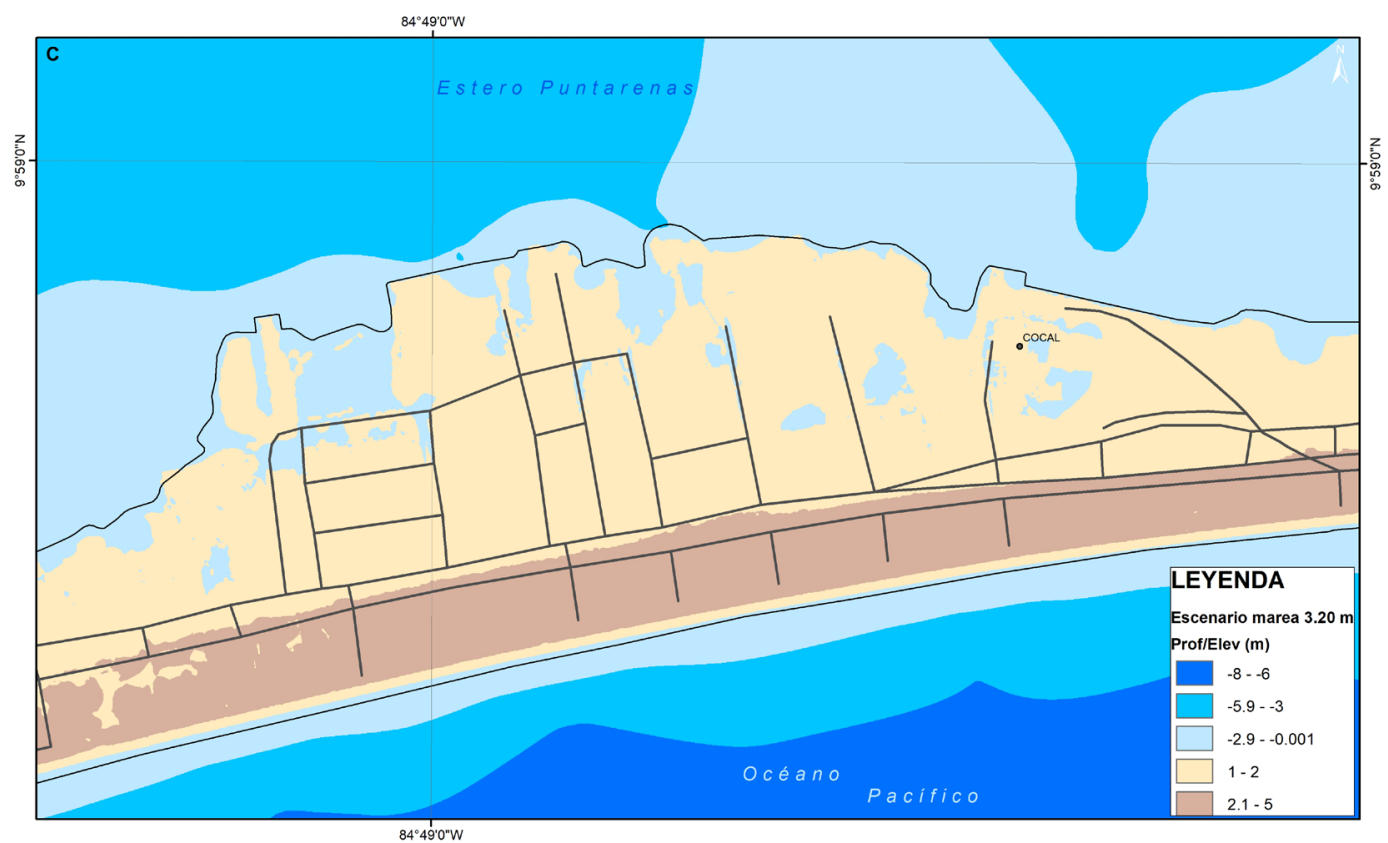


Lizano Araya, MA. y Lizano Rodríguez, O. (2020): "Escenarios ante el aumento del nivel del mar por cambio climático para la localidad del Cocal, Puntarenas, Costa Rica", GeoFocus, revista Internacional de Ciencia y Tecnología de la Información Geográfica, 26, 3-20. http://dx.doi.org/10.21138/GF.656
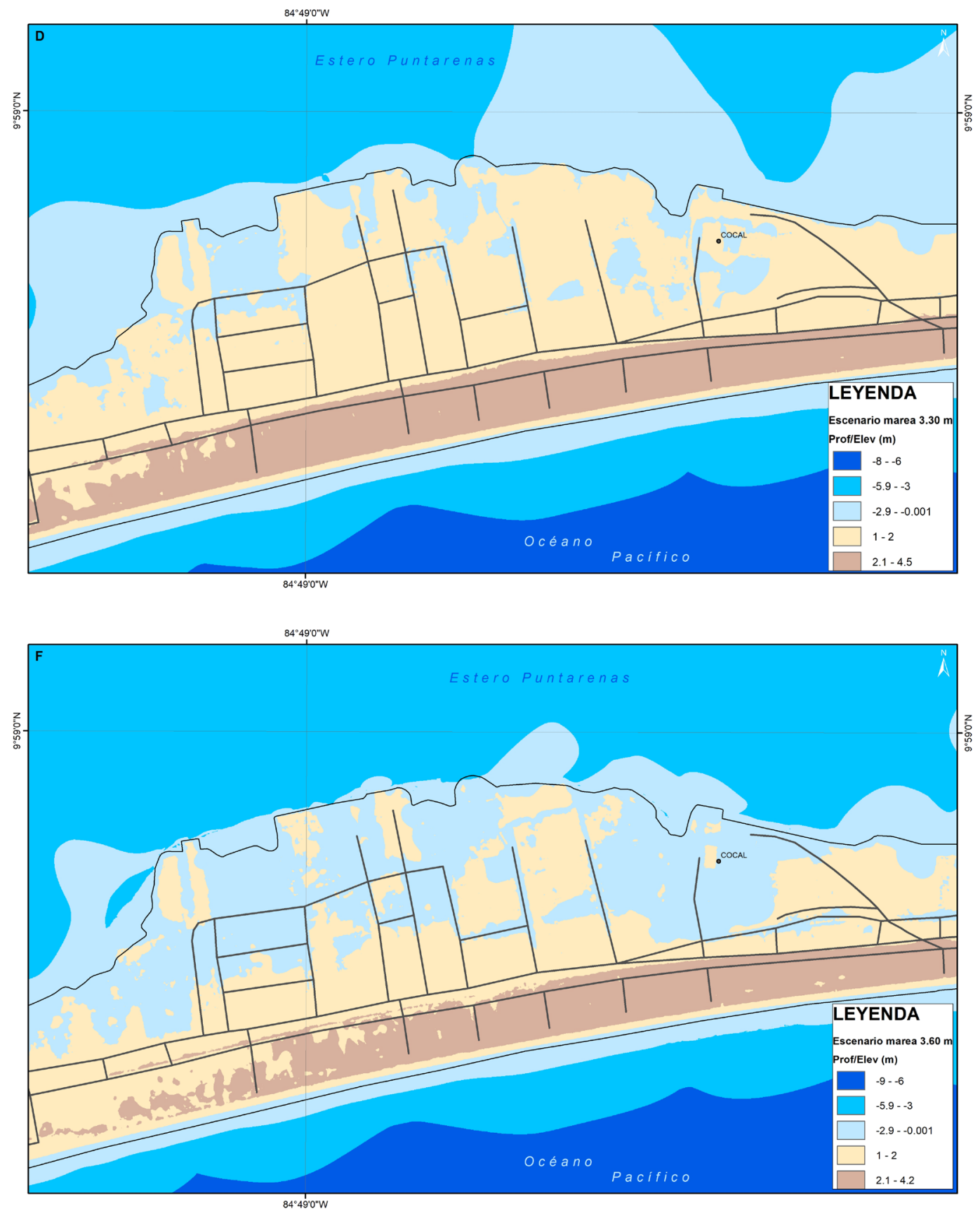
Lizano Araya, MA. y Lizano Rodríguez, O. (2020): "Escenarios ante el aumento del nivel del mar por cambio climático para la localidad del Cocal, Puntarenas, Costa Rica", GeoFocus, revista Internacional de Ciencia y Tecnología de la Información Geográfica, 26, 3-20. http://dx.doi.org/10.21138/GF.656

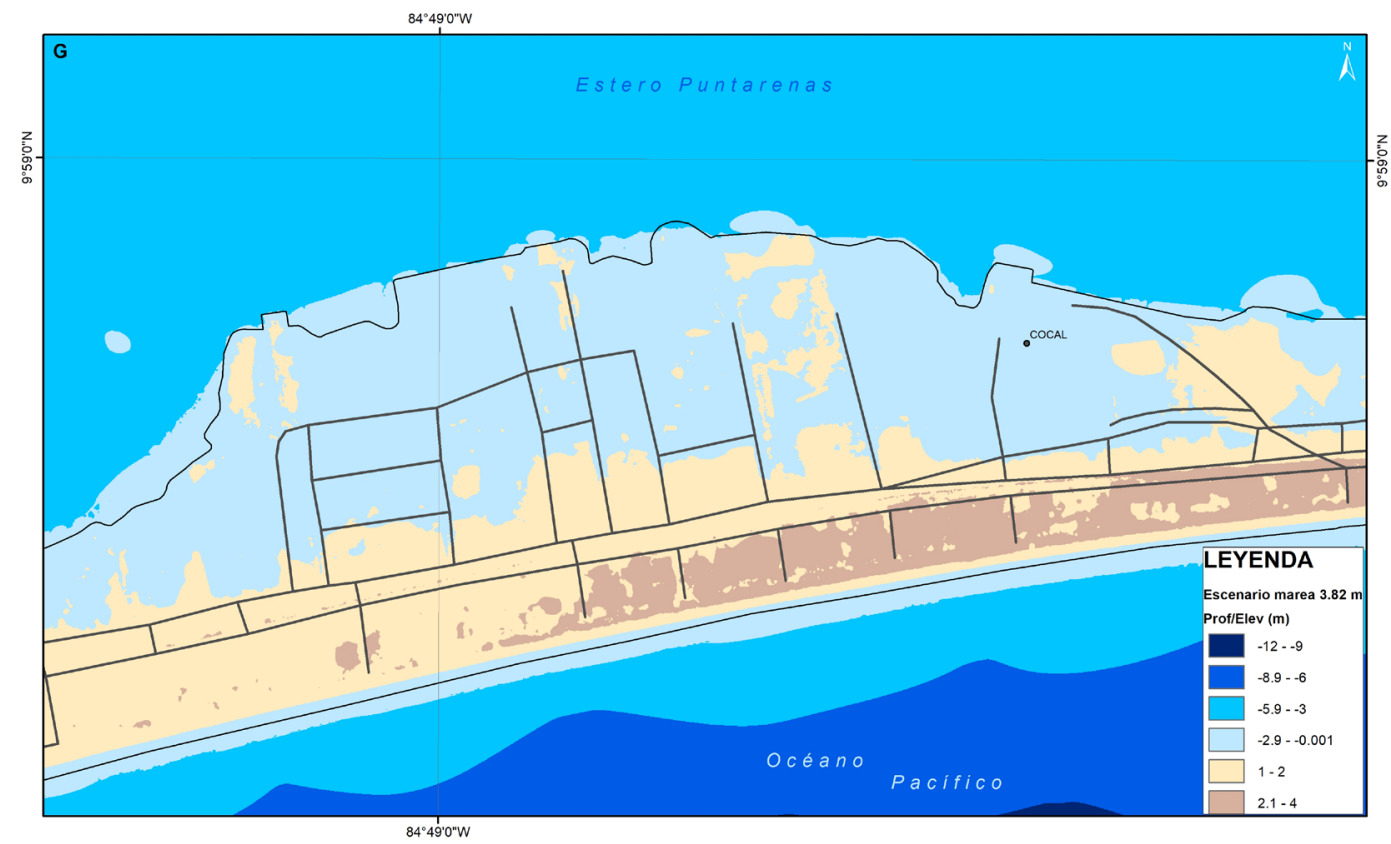

Fig. 5. Niveles de inundación por mareas para el Cocal de Puntarenas a partir de marea 3 m con aumento relativo

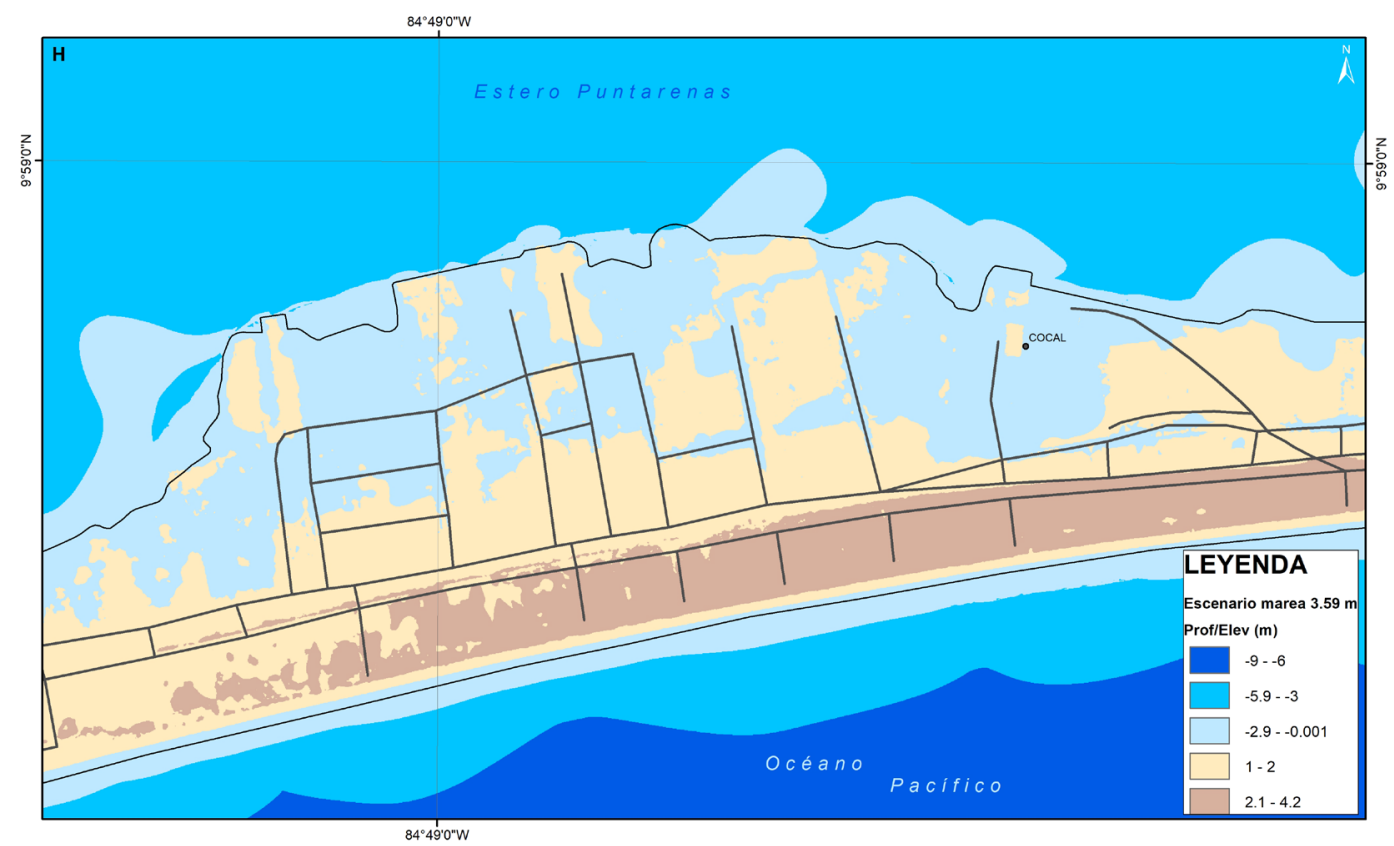


Lizano Araya, MA. y Lizano Rodríguez, O. (2020): "Escenarios ante el aumento del nivel del mar por cambio climático para la localidad del Cocal, Puntarenas, Costa Rica", GeoFocus, revista Internacional de Ciencia y Tecnología de la Información Geográfica, 26, 3-20. http://dx.doi.org/10.21138/GF.656
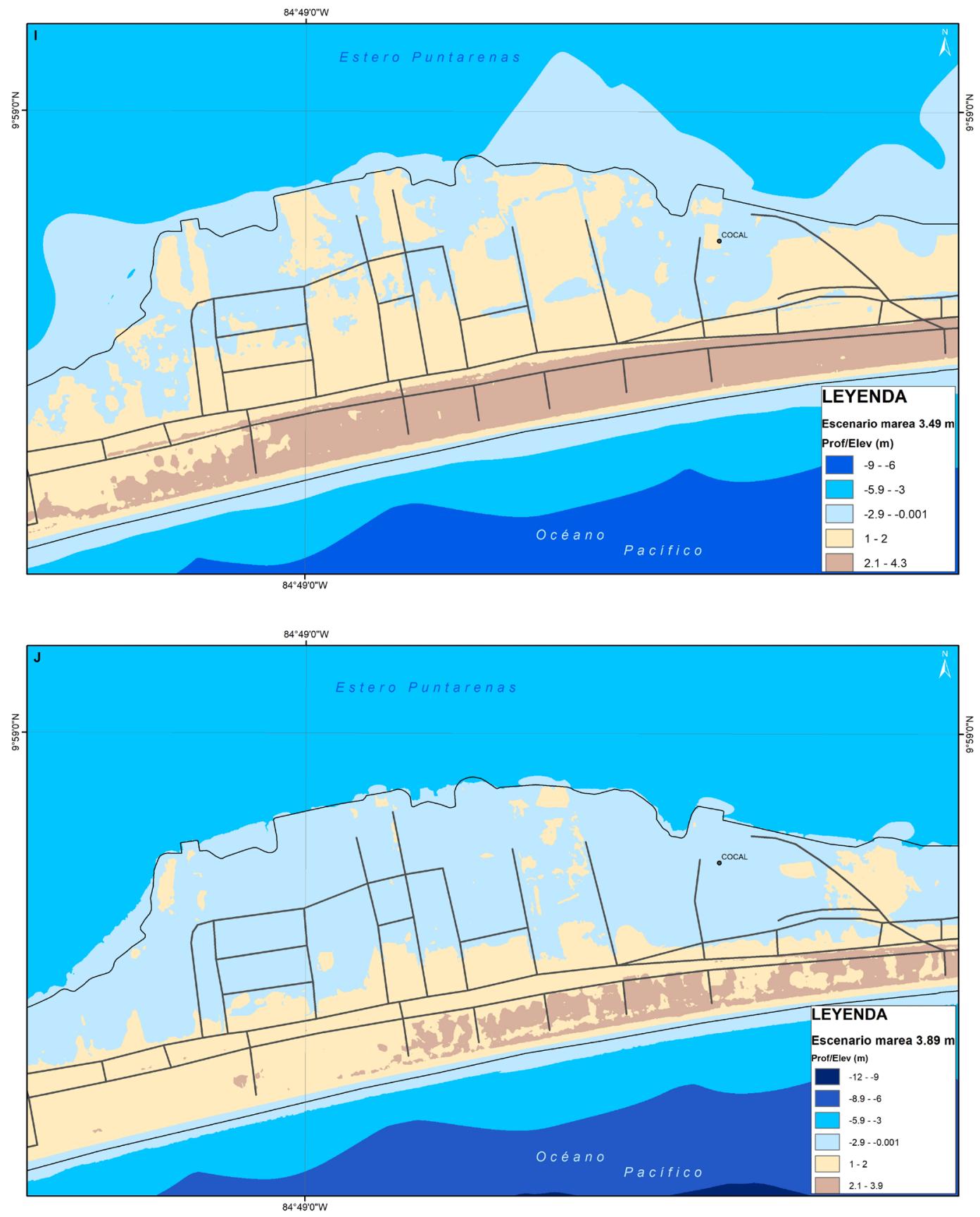
Lizano Araya, MA. y Lizano Rodríguez, O. (2020): "Escenarios ante el aumento del nivel del mar por cambio climático para la localidad del Cocal, Puntarenas, Costa Rica", GeoFocus, revista Internacional de Ciencia y Tecnología de la Información Geográfica, 26, 3-20. http://dx.doi.org/10.21138/GF.656

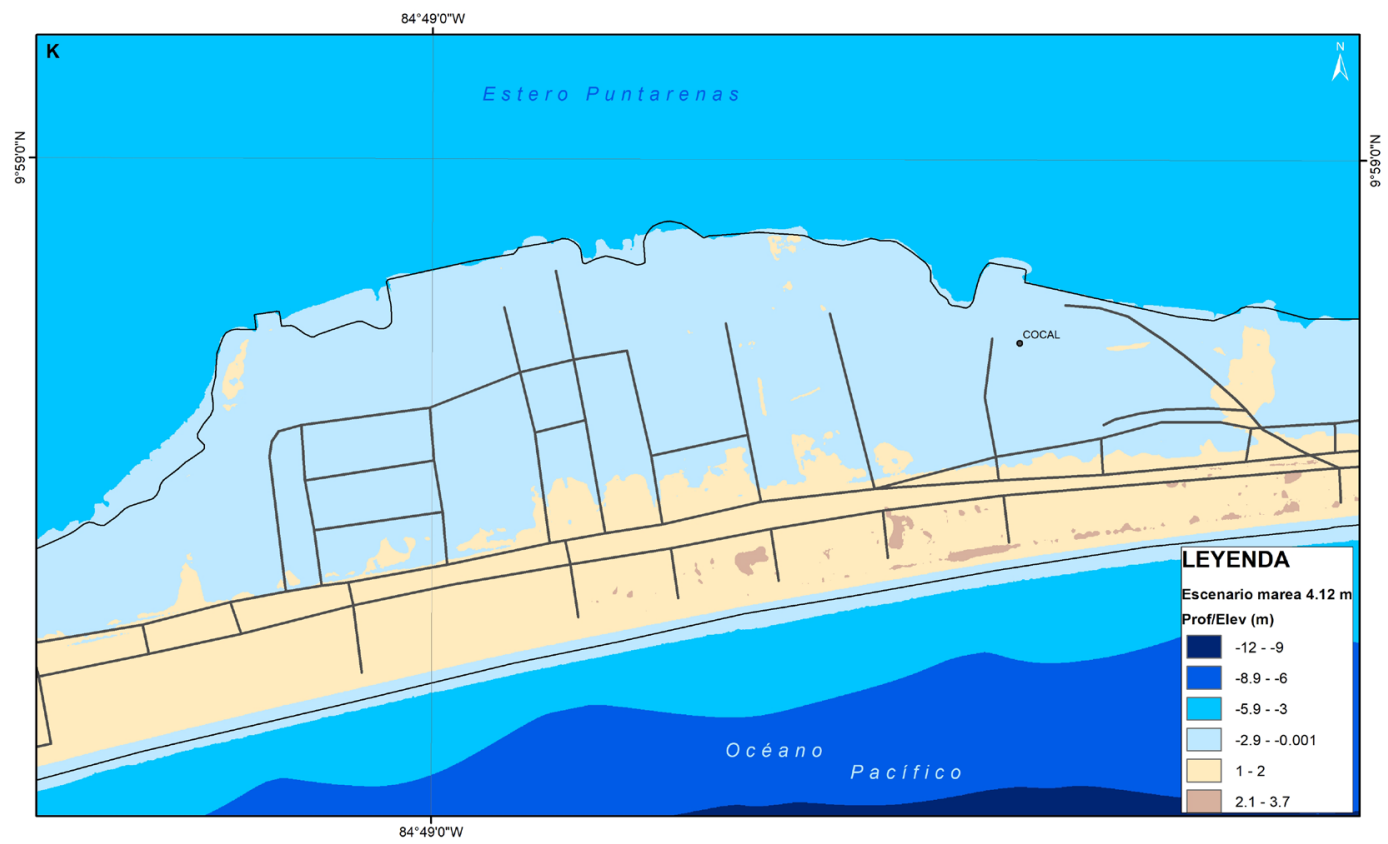

Fig. 6. Niveles de inundación por mareas para el Cocal de Puntarenas a partir de marea 3.29 m con aumento relativo. Aclaración. Las letras que aparecen en la esquina superior izquierda corresponden a los distintos escenarios tomando como base la marea de $3.29 \mathrm{~m}$ y su aumento relativo.

Como lo muestra la totalidad de escenarios del A al K, la inundación comienza por el estero, a partir de mareas que superen $\operatorname{los} 2.95 \mathrm{~m}$, donde se aprecia la inundación en el sector del Cocal. Para mareas superiores a los $3 \mathrm{~m}$ comienza una inundación progresiva que aumenta de acuerdo con el nivel de marea, como se aprecia claramente en todas las figuras mostradas anteriormente. Los escenarios G y K muestran los peores escenarios de inundación hacia el año 2100. En todos los escenarios generados, el impacto no se da por el frente costero debido a la topografía del terreno y la pendiente, siendo la línea del tren uno de los terrenos más altos al frente de la punta de Puntarenas cuya pendiente decrece hacia el sector del estero. De esta manera, cuando el nivel del mar comienza a ascender paulatinamente, la marea entra por el estero y comienza a generar problemas de inundación en la comunidad del Cocal y en otros sitios de Puntarenas. Si se diera una combinación de otros fenómenos como el Niño, Apilamiento del Oleaje u otros fenómenos hidrometeorológicos, los efectos indicados en esta investigación se agravarían con el consecuente problema para la comunidad del Cocal.

\section{Discusión y valoración de hallazgos}

El ascenso del nivel del mar por cambio climático supondrá un problema para la comunidad del Cocal y Puntarenas en el corto plazo (30 años) (IPCC, 2007, 21). Desde la mitad del siglo pasado el ritmo del aumento ha sido muy superior a la media de los dos milenios que le precedieron 
Lizano Araya, MA. y Lizano Rodríguez, O. (2020): "Escenarios ante el aumento del nivel del mar por cambio climático para la localidad del Cocal, Puntarenas, Costa Rica", GeoFocus, revista Internacional de Ciencia y Tecnología de la Información Geográfica, 26, 3-20. http://dx.doi.org/10.21138/GF.656

(IPCC, 2014, 46). Lo anterior queda reflejado en las estimaciones realizadas a partir de los datos mostrado en la tabla 1 que mostrarían un ascenso significativo del nivel del mar, cuya causa directa está asociada con el aumento de la temperatura global, lo cual ha originado y está causando un derretimiento acelerado de los glaciares en muchas partes del mundo, con el consecuente problema de un mayor aporte de agua en todos los océanos (Lizano, 2013, 7). Lo anterior ha generado un aumento creciente del nivel del mar y se mantendrá hacia posteriores años; como se ha valorado en este documento. En consecuencia, se debe ir pensando en propuestas de mitigación, para prevenir eventos y desastres mayores en el área en mención.

Una peculiaridad obtenida a partir de los fenómenos modelados es que la inundación en la zona del Cocal comienza por el estero de Puntarenas. Esta zona se caracteriza por presentar las menores elevaciones, lo que provoca que, durante un ascenso superior a los $2.95 \mathrm{~m}$ paulatino o extraordinario, las inundaciones se manifiesten con mayor frecuencia hacia los asentamientos que colindan con estas zonas. Las zonas que no presentan afectación alguna se dan en el frente costero, a lo largo de la línea del tren (hacia el Sur), así como lo que colinda alrededor de la Ruta 23 (ingreso a Puntarenas).

Otro punto por valorar se da a causa de los problemas que acarreará el máximo aumento del nivel del mar $(0.60 \mathrm{~cm}-0.82 \mathrm{~cm})$, pues bajo estos escenarios el incremento no implicará un tipo de marea alta y marea baja. Se espera que a partir del año 2090 los sectores circundantes al Poblado del Cocal, estarán inundados. Posterior al año 2100 es muy factible que los sectores donde tradicionalmente se ha inundado por alguna marea máxima u otro evento poco tradicional, estén completamente inundados bajo una capa de agua que oscilará entre los $0.90 \mathrm{~cm}$ o más centímetros iniciando al sector oeste de la comunidad del Cocal y siguiendo longitudinalmente hacia el Este.

Según el software Tides \& Currents for Windows (Lizano, 2006, 53), la mayor marea astronómica, para Puntarenas es de 3,29 m. En un estudio adicional de las mareas altas de 20 años de esta zona, se demuestra que la frecuencia de superar una marea de $3 \mathrm{~m}$ para el sitio es de 42.3 veces/año. Para el año 2050 con un escenario adicional de $0.30 \mathrm{~m}$, la frecuencia de tener niveles del mar con valores mayores o iguales a $3 \mathrm{~m}$ sería de 391 veces/año. Para los demás escenarios (2070 y 2100), la probabilidad de tener niveles del mar superiores a ellos, serán todos los días para las zonas señaladas en este trabajo.

Otro de los fenómenos que puede conllevar algunos tipos de problemas para las poblaciones, sería el causado por las tormentas lejanas y/o locales (Lizano, 2010), que provocan el apilamiento del oleaje no modelado en este estudio, pero que al sumarse con un período de marea alta podría incidir en algunas inundaciones en partes importantes cercanas más allá de los escenarios aquí planteados para la zona del Cocal. Bajo crecientes aumentos del nivel del mar, el oleaje se constituye en el factor más dinámico y una fuente de energía suficiente para causar impacto costero en estas áreas, hasta el grado de favorecer problemas de erosión más acrecentados en las zonas del estudio, aumentando los que ya tiene (Lizano, 2013, 19). 
Lizano Araya, MA. y Lizano Rodríguez, O. (2020): "Escenarios ante el aumento del nivel del mar por cambio climático para la localidad del Cocal, Puntarenas, Costa Rica", GeoFocus, revista Internacional de Ciencia y Tecnología de la Información Geográfica, 26, 3-20. http://dx.doi.org/10.21138/GF.656

\section{Conclusiones}

Por el grado de importancia que tiene este tema, en el cercano, mediano y largo plazo, hacia la comunidad del Cocal, es que las autoridades respectivas deberían invertir en equipo tecnológico que permita cuantificar estos escenarios de manera más precisa. Para ello se recomienda instalar un mareógrafo que permita medir y obtener patrones sobre el nivel del mar, con el fin de tener un registro continuo de esas variaciones, lo cual puede ayudar a prevenir y mitigar posibles amenazas, así como otros que puedan ocurrir y que no se encuentran citados en este documento.

El panorama mostrado en este documento se puede tornar difícil si a parte del aumento del nivel del mar se consideran otras variables o fenómenos que puedan presentarse para un día particular como el fenómeno de El Niño, alta precipitación o un tsunami, y que en un mismo día estos pueden superponerse, pues implicarían aumentos importantes en el nivel del mar, al punto que podrían ocasionar inundaciones aún mayores a las mostradas en este documento. A partir de lo anterior, las autoridades correspondientes deberían tener algún plan de acción o de emergencia para eventos que produzcan impacto costero, o construir la infraestructura necesaria para proteger la punta, y comenzar a trabajar en la posible reubicación de toda la población o proponer nuevos códigos constructivos por este tipo de fenómenos.

Es meritorio aclarar que en este estudio se han tratado los distintos niveles mareográficos como una superposición de niveles pasivos simplemente, sin tomar en cuenta que la dinámica de ciertas componentes, como las corrientes y el oleaje, tienen la capacidad de modificar la geomorfología costera en un corto tiempo ante eventos extraordinarios. Esto puede desequilibrar el sistema para siempre antes de lo imaginable si no se toman las medidas respectivas pronto, pues como lo señala Lizano (2011), ya el aumento del nivel del mar es una realidad en nuestras costas a la vez si sucede lo mencionado sería bueno utilizar técnicas como el Lidar o la Fotogrametría con vehículos aéreos no tripulados para obtener cartografía a detalle que permita nuevamente modelar los escenarios para una toma de decisiones anotadas en este estudio.

El otro componente que no se incluyó en este estudio, es el aumento el nivel del mar por un tsunami. El tsunami reciente de Japón (10 de marzo del 2011) produjo un aumento del nivel del mar en Quepos de $18 \mathrm{~cm}$ según datos del Centro de Alerta de Maremotos del Pacífico Consultado el 20 de Julio de 2020 (http://ptwc.weather.gov). Hay simulaciones de Ortíz et al. (2001) de tsunamis históricos con ondas de $3.5 \mathrm{~m}$ de altura en Puntarenas. De manera que los escenarios incluidos aquí, también sirven para sobreponer este componente a las condiciones de la marea existente en el momento de arribo de un tsunami, y constituye una herramienta válida para que las autoridades respectivas avalúen las condiciones del nivel del mar ante un evento de este tipo en el Océano Pacífico.

Es de suma importancia que la variable de aumento del nivel del mar se incluya en la elaboración de los planes reguladores costeros, con el fin de que sirva como un instrumento adecuado para el ordenamiento territorial y la planificación urbana por parte de las instituciones pertinentes, mismo que debería ser de acatamiento obligatorio para las Municipalidades que en su espacio, cuenten con el componente costero. 
Lizano Araya, MA. y Lizano Rodríguez, O. (2020): "Escenarios ante el aumento del nivel del mar por cambio climático para la localidad del Cocal, Puntarenas, Costa Rica", GeoFocus, revista Internacional de Ciencia y Tecnología de la Información Geográfica, 26, 3-20. http://dx.doi.org/10.21138/GF.656

\section{Agradecimientos}

A los estudiantes Andrés Vargas Mena, Linnette Ceciliano Calderón y Jennifer Fernández Garro, estudiantes de Geografía de la Universidad de Costa Rica, por la colaboración en la elaboración de este artículo. A la Vicerrectoría de Investigación por apoyo a través del proyecto No. B5267.

\section{Referencias bibliográficas}

Bedia Jiménez, J. 2004. Creación de un modelo digital de elevación para la predicción de futuros escenarios de inundación en el estuario del Río Loughor (Sur de Gales). Proyecto fin de carrera Bachelor (Honours) Environmental Science Wolverhampton University, School of Applied Sciences (U.K.) $<$ http://www.joaquinbedia.es/MDT.htm/> (actualizado julio 2004, consultado 6 de abril del 2009).

CEPAL. 2012. Efectos del Cambio Climático en la costa de América Latina y el Caribe: Impactos. División de Desarrollo Sostenible y Asentamientos Humanos de la CEPAL. Naciones Unidas, Santiago, Chile. 120.

Cheng, L; Zhu, J; Abraham, J; Trenberth, K; Fasullo, J; Zhang, B; Yu, F; Wan, L; Chen, X, y Z, Song. 2018. Continues Record Global Ocean Warming. Advances in Atmospheric Sciences. 36(3), 249-252, https://doi.org/10.1007/s00376-019-8276-x

Diaz Andrade, J. 1999. Determinación de las zonas de riesgo ante un ascenso del nivel del mar: Punta Morales-Tárcoles (Informe Final). MINAE-IMN. San José, Costa Rica, 59.

Dawahidi, T; Ibarra, D; y F, Gomariz. 2019. Estimación de la subida del nivel del mar por efecto del Cambio Climático mediante datos de mareógrafos y la serie de satélite NASA TopexPoseidon/Jason. El caso de Valencia. Anales de Geografía de la Universidad Complutense. Ediciones Complutense. ISSN: 0211-9803. http://dx.doi.org/10.5209/AGUC.64676.

IPCC. 2001. Cambio climático 2001: Informe de síntesis, Resúmenes de los Grupos de Trabajo. IPCC, Ginebra, Suiza, 86.

IPCC. 2007. Cambio climático 2007: Informe de síntesis. Informe del Grupo Intergubernamental de Expertos sobre el Cambio Climático [Equipo IPCC. y Pachauri, R y L, Meyer. (directores de la publicación)]. IPCC, Ginebra, Suiza, 114.

IPCC, 2014: Cambio climático 2014: Informe de síntesis. Contribución de los Grupos de trabajo I, II y III al Quinto Informe de Evaluación del Grupo Intergubernamental de Expertos sobre el Cambio Climático [Equipo principal de redacción, R.K. Pachauri y L.A. Meyer (eds.)]. IPCC, Ginebra, Suiza, 157 págs.

Kamphuis William, J 2000. Introduction to Coastal Engineering and Management. Advanced Series on Ocean Engineering- Volume 16. Word Scientific. New Jersey, 437.

Lambrechts, C. 2007. Global Outlook for Ice \& Snor. Heather Main (lead), UNEP/GRID-Arendal. Noruega, 235. 
Lizano Araya, MA. y Lizano Rodríguez, O. (2020): "Escenarios ante el aumento del nivel del mar por cambio climático para la localidad del Cocal, Puntarenas, Costa Rica", GeoFocus, revista Internacional de Ciencia y Tecnología de la Información Geográfica, 26, 3-20. http://dx.doi.org/10.21138/GF.656

Lizano Araya, M; Lizano Rodríguez, O.2010.Creación de escenarios de inundación en la Ciudad de Puntarenas ante el aumento del nivel del mar. InterSedes. Vol. XI. (21-2010) 215-229. ISSN: 22152458

Lizano Rodríguez, O.G. 1997. Las Mareas Extraordinarias de 1997 en la Costa del Pacífico de Costa Rica. Top. Meteor. Oceanogr., 4 (2), 169-179.

Lizano Rodríguez, O.G. \& Salas Gonzáles, D. 2001. Variaciones geomorfológicas en los últimos 50 años de la Isla Damas, Quepos, Costa Rica. Rev. Biol. Trop., 2, 171-177.

Lizano Rodríguez, O.G. 2006. Algunas Características de las Mareas en la Costa Pacífica y Caribe de Centroamérica. Ciencia y Tecnología, 51-64.

Lizano Rodríguez, O.G. 2007. Climatología del viento y el oleaje frente a las costas de Costa Rica. Ciencia y Tecnología. Volumen 25 (1 y 2), 43-56

Lizano Rodríguez, O.G. 2010. Batimetría, modelo de elevación digital y sus aplicaciones. Revista AZIMUTH 10 DIGITAL, 18-21.

Lizano Rodríguez, O.G. 2013. Erosión en las playas de Costa Rica, incluyendo la Isla del Coco. InterSedes, XIV (17): 6-27.

Nerem Steven, R; Beckley, D; Fasullo, J; Hamlington,, D; Masters, D y G, Mitchum. 2018 Climate change driven accelerated sea-level rise detected in the altimeter era. National Academy of Sciences. vol. 115 no. 9 2022-2025

Ortiz, M. Fernández, M. y W, Rojas. 2001: Riesgo de inundación por tsunamis en Puntarenas, Costa Rica. GEOS, V. 21, N 2: 108-113.

Otto-Bliesner, J. Marshal, S. Overpeck, .J. y G, Miller. 2006. Simulating Arctic Climate Warmth and Icefield Retreat in the Last Interglaciation. Science. 311, 1751-1753.

PNUMA. 2013. GEO-5 Perspectivas del Medio Ambiente Mundial. Medio ambiente para el futuro que queremos. Programa de las Naciones Unidas para el Medio Ambiente. 480.

Ramos, R. Zavala, J. Gama, C. Lilia, Pech. Pool, D. y M.A, Ortiz. 2016. Indicadores geomorfológicos para evaluar la vulnerabilidad por inundación ante el ascenso del nivel del mar debido al cambio climático en la costa de Tabasco y Campeche, México. Boletín de la Sociedad Geológica Mexicana, 68(3), 581-598. Recuperado en 22 de julio de 2020, de http://www.scielo.org.mx/scielo.php?script=sci_arttext\&pid=S1405-

33222016000300581\&lng=es\&tlng=en.

Van der Meulen, F. Witter, J. y S, Marens. 1991. The use of a GIS in Assessing the Impacts of SeaLevel Rise on Nature Conservation along the Dutch Coast. Landscape Ecology 6 (1/2), 105-113. 